\title{
Utilidad diagnóstica de los nódulos de Osler en la endocarditis infecciosa en usuarios de drogas por vía parenteral (UDVP)
}

\author{
F. J. ESPINOSA PARRA, J. M. RAMOS RINCÓN, F. HERRERO HUERTA, \\ L. PRETEL SERRANO, A. A. LORENZO
}

Servicio de Medicina Interna. Hospital General Universitario J.M. Morales Meseguer. Murcia

\author{
DIAGNOSTIC UTILITY OF OSLER'S NODES IN THE INFECTIVE \\ ENDOCARDITIS IN INTRAVENOUS DRUG USERS
}

\section{RESUMEN}

Fundamento: Se describen los datos clínicos, microbiológicos e histológicos de cinco episodios de endocarditis infecciosa (EI) con nódulos de Osler en usuarios de drogas por vía parenteral (UDVP).

Pacientes y métodos: Se han estudiado de forma prospectiva 43 casos de EI en UDVP. En 4 pacientes se realizó punción aspiración y en otro biopsia de un nódulo de Osler, con tinción de Gram y cultivo de la muestra.

Resultados: De los 43 episodios de EI 33 fueron derechas, 9 izquierdas y 1 mixta. Cinco de los $10(50 \%)$ pacientes con endocarditis izquierda o mixta presentaron nódulos de Osler, pero no se encontraron en ninguno de los pacientes con EI derecha. En todas las muestras tomadas por punción aspiración se observaron cocos grampositivos en racimos en la tinción de Gram y se obtuvo crecimiento de Staphylococcus aureus con el mismo antibiotipo que los aislados en los hemocultivos. En el único caso en el que se realizo biopsia del nódulo se apreciaba trombos sépticos en la microcirculación.

Conclusiones: La tinción de Gram y el cultivo del material aspirado de los nódulos de Osler tiene una alta rentabilidad en el diagnóstico etiológico de la EI en los usuarios a drogras por vía parenteral. La presencia de nódulos de Osler en un paciente con EI nos debe sugerir que la localización es izquierda. Estos datos sugieren que los nódulos de Osler, en la EI por S. aureus en los UDVP se origina como consecuencia de embolismos sépticos microvasculares.

PALABRAS CLAVE: Endocarditis infecciosa. Usuarios de drogas por vía parenteral. Nódulos de Osler.

\section{ABSTRACT}

Background: The objective of study is to describe of clinic, microbio logical and histological data of five cases of infective endocarditis (IE) with Osler's nodes in intravenous drug users .

Patients y methods: Prospectively, 43 cases of IE in intravenous drugs users was revised. In 4 patients, a aspirate puncture of Osler's node was performed and in one patient a biopsy of Osler's node was done with Gram's stain and culture of specimen.

Results: From 43 episodes of IE, 33 were right-side IE, 9 left-side y 1 right and left side. No patients with right-side IE presented Osler's nodes, however five of 10 (50\%) patients with left-side endocarditis. In all of cases grampositive cocci were observed in Gram's strain and Staphylococcus aureus growth on culture of lesion with the same antibiotype than isolated from blood culture. One case a cutaneous biopsy was performed, and inflammatory infiltrate with necrosis was found.

Conclusions: The Gram's strain and culture of specimen aspirated from Osler's nodes were of high utility in the diagnosis of IE in intrave nous drugs users. The presence of Osler's nodes in a patient with infecti ve endocarditis must be suggest that the location in left-side. These data suggest that Osler's nodes in infective endocarditis by S. aureus in intra venous drugs users was originated by microvascular septic emboli.

KEY WORS: Infective endocarditis. Intravenous drugs users. Osler's nodes.

Espinosa Parra FJ, Ramos Rincón JM, Herrero Huerta F, Pretel Serrano L, Lorenzo AA. Utilidad diagnóstica de los nódulos de Osler en la endocarditis infecciosa en usuarios de drogas por vía parenteral (UDVP). An Med Interna (Madrid) 2002; 19: 299-301.

\section{INTRODUCCIÓN}

Las manifestaciones cutáneas de la endocarditis infecciosa incluyen petequias, hemorragias subungueales, nódulos de Osler y lesiones de Janeway (1). Los nódulos de Osler son lesiones cutáneas nodulares eritematosas dolorosas, localizadas preferentemente en el pulpejo de los dedos. La naturaleza histológica de estas lesiones es dudosa (2,3). Clásicamente se consideraban lesiones vasculíticas mediadas inmunológicamente sin formación de microabscesos,concepto que se sigue empleando en los libros de texto (1) y en los nuevos criterios diagnósticos de endocarditis de Durack y cols. (4). En la literatura más reciente se recoge que las biopsias de los nódulos de Osler se observan datos histológicos de microembolismos con cultivo positivo de las mismas $(2,3)$. El objetivo de este trabajo es describir los datos clínicos, microbiológicos e

Trabajo aceptado: 14 de diciembre de 2000

Correspondencia: José Manuel Ramos Rincón. Avda. Ancha de Castelar, 79 6-B. 03690 San Vicente del Raspeig. Alicante. 
histológicos de cinco episodios endocardits infecciosa (EI) con nódulos de Osler en usuarios de drogas por vía parenteral (UDVP).

\section{CASOS APORTADOS}

Se evaluaron prospectivamente 43 episodios de endocarditis infecciosa en UDVP ingresados desde octubre de 1986 hasta diciembre de 1994 en el Hospital Santa María del Rosell de Cartagena (Murcia). Un autor (FJEP) ha sido durante este periodo responsable directo de los pacientes con EI captados prospectivamente. Los criterios diagnósticos de EI fueron los propuestos por Von Reyn y cols. (5), modificados para incluir los datos ecocardiograficos como un nuevo criterio. La existencia de drogadición por vía parenteral se basó en la anamnesis y/o presencia de estigmas de venopunción repetidas (signos de punción venosa y venas superficiales induradas, trombosadas y pigmentadas). La localización de la EI se estableció mediante el estudio ecocardiográfico o por datos clínicos/radiológicos.

Se consideró nódulos de Osler a las lesiones nodulares, dolorosas, rojas o purpúricas localizadas en los pulpejos de los dedos y/o eminencia tenar o hipotenar. Sobre las lesiones se realizó una punción aspirativa con aguja de $10 \mathrm{G}$ y pistola de aspiración Kameco. En las muestras obtenidas se realizó tinción de Gram y cultivo. En un caso se realizó biopsia del nódulo para estudio histológico y cultivo.

De los 43 episodios de EI en UDVP, 33 fueros derechas, 9 izquierdas y 1 mixta. Cinco de los $10(50 \%)$ pacientes con endocarditis izquierda o mixta presentaron nódulos de Osler, pero estos no se encontraron en ninguno de los pacientes con EI derecha. La tabla I resume las características clínicas y microbiológicas de los cinco episodios. Cuatro paciente eran varones y uno mujer, con una edad comprendida entre los 23 y 35 años. La duración de los síntomas de EI en el momento de la toma de la muestra del nódulo de Osler fue de 4 a 12 días (media de 7,2 días). Los cinco pacientes tenían endocarditis mitral, además uno de ellos mostraba también vegetaciones en válvula aórtica y otro en la válvula tricuspídea. Los nódulos de Osler se localizaban en todos los casos en los dedos de los pies y en 4 de ellos también en los dedos de las manos. En los 4 casos en los que se realizó la tinción de Gram del aspirado del nódulo de Osler se observaron cocos grampositivos en racimos. En los 5 pacientes se obtuvo crecimiento de Staphylococcus aureus en las muestras proce- dentes de los nódulos de Osler. El mismo microorganismo también creció en los hemocultivos de todos los pacientes. En el único caso en el que se realizó biopsia de la lesión se apreciaba en la dermis un infiltrado inflamatorio con necrosis y en las arterias de mediano calibre de la unión dermo-hipodérmica y en los capilares se observaron trombos con cocos grampositivo en racimo en su interior, identificados en el cultivo como $\mathrm{S}$. aureus.

\section{DISCUSIÓN}

Las lesiones cutáneas en forma de nódulos de Osler y/o lesiones de Janewey están presentes en el 3-6\% las series recientes de EI (6-8), porcentaje claramente inferior al recogido en la era preantibiótica (aproximadamente 40-80\%)(9-10). Los nódulos de Osler y las lesiones de Janeway son distintas clínicamente (3), las primeras son lesiones nodulares eritematosas y dolorosas localizadas en los pulpejos de los dedos y las segundas son lesiones hemorrágicas indoloras localizadas en palmas y plantas (11).

En 1893 Osler, cuando describió los nódulos que posteriormente llevarían su nombre, postuló la posibilidad de que fueran debidos a diminutos focos embólicos (12). Posteriormente, en las sucesivas aportaciones histológicas de los nódulos de Osler recogidas en la literatura se describen como fenómenos embólicos con reacción inflamatoria debida a un estímulo desconocido. Así, mientras Merklen y Wolf (13) en 1928 describíeron infiltrado de células inflamatorias perivascular en los nódulos de Osler, Lian y cols. (14), en 1929, encontraron infiltrado de neutrófilos y proliferación de células endoteliales, y Cornil y cols. (15), en 1939, no hallaron microorganismos en las lesiones. Posteriormente, Von Gemminger y Winkelmann (16) vuelven a encontrar en los nódulos de Osler focos de embolismo estéril.

En 1976, Alpert y cols. (17) estudiaron en las biopsias de los nódulos de Osler y en los hallazgos histológicos encuentran microabscesos en la dermis papilar y microembolos en la dermis profunda. Estas lesiones se asemejan a los datos histológicos descritos por Kerr de las lesiones de Janeway (18).

TABLA I

CARACTERÍSTICAS CLÍNICO -M ICRO BIO LÓ GICAS DE LOS CINCO PACIENTES CON ENDOCARDITIS INFECCIOSA Y NÓ DULOS DE OSLER

\begin{tabular}{|c|c|c|c|c|c|c|c|c|c|}
\hline Paciente & $\begin{array}{c}\text { Edad } \\
\text { (años) }\end{array}$ & Sexo & $\begin{array}{l}\text { Válvula } \\
\text { afectada }\end{array}$ & $\begin{array}{l}\text { M icroorganismos } \\
\text { en los } \\
\text { hemocultivos }\end{array}$ & $\begin{array}{l}\text { Localización } \\
\text { nódulos de } \\
\text { O sler }\end{array}$ & $\begin{array}{l}\text { Tinción de } \\
\text { Gram }\end{array}$ & $\begin{array}{c}\text { M icroorganismos } \\
\text { en el } \\
\text { cultivo }\end{array}$ & Biopsia & $\begin{array}{c}\text { Duración } \\
\text { de la El } \\
\text { (días) }\end{array}$ \\
\hline 1 & 25 & $\mathrm{H}$ & M itral & S. aureus & $\begin{array}{l}\text { Dedos de las } \\
\text { manos y pies }\end{array}$ & CG+ & S. aureus & NR & 4 \\
\hline 2 & 23 & $\mathrm{H}$ & $\begin{array}{c}\text { Mitral y } \\
\text { Tricúspide }\end{array}$ & S. aureus & $\begin{array}{l}\text { Dedos de las } \\
\text { manos y pies }\end{array}$ & CG+ & S. aureus & NR & 10 \\
\hline 3 & 35 & $\mathrm{H}$ & M itral & S. aureus & $\begin{array}{l}\text { Dedos de los } \\
\text { pies }\end{array}$ & CG+ & S. aureus & NR & 12 \\
\hline 4 & 35 & $M$ & M itral & S. aureus & $\begin{array}{l}\text { Dedos de las } \\
\text { manos y pies }\end{array}$ & CG+ & S. aureus & NR & 4 \\
\hline 5 & 31 & $\mathrm{H}$ & $\begin{array}{l}\text { Mitral y } \\
\text { Aórtica }\end{array}$ & S. aureus & $\begin{array}{l}\text { Dedos de las } \\
\text { manos y pies }\end{array}$ & NR & S. aureus & $\begin{array}{l}\text { Abscesos } \\
\text { dérmicos } \\
\text { múltiples }\end{array}$ & 5 \\
\hline
\end{tabular}

El: endocarditis infecciosa, NR: no realizado; $\mathrm{H}$ : hombre, $\mathrm{M}$ : mujer; $\mathrm{CG}+$ : cocos grampositivos en tetradas o racimos. 
Por todo ello Alpert y cols. sugieren que los nódulos de Osler y las lesiones de Janeway, distintas clínicamente, son similares en la patogenia como consecuencia de émbolos sépticos (17). Otros autores posteriores defienden la misma postura patogénica (2,3). El único de nuestros casos en el que disponemos de biopsia apoya los hallazgos de Alpert y cols. del origen microembólico séptico de los nódulos de Osler. A pesar de estos hallazgos, se sigue considerando a los nódulos de Osler como lesiones mediadas inmunológicamente. En este sentido los nuevos criterios diagnósticos de endocarditis de Durack y cols. (4) se siguen considerando que los nodulos de Osler, las manchas de Roth y la glomerulonefritis secundaria a EI están mediadas inmunológicamente.

Los nódulos de Osler son lesiones cutáneas no patognomónicas de endocarditis y se han descrito también en la fiebre tifoidea, lupus eritematosos sistémico, gonococemia y endocarditis trombótica no bacteriana $(9,19)$, pero son lesiones que deben hacer sospechar al clínico la presencia de una EI. Así, se han descritos casos en los que los nódulos de Osler han sido el signo físico que ha dirigido el diagnóstico hacia una endocarditis $(20,21)$, en alguna ocasión por microorganismos que no crecen en los hemocultivos como Chlamydia pneumo niae (22).

En los cultivos de los nódulos de Osler se han aislado los mismos microorganismo que en los hemocultivos simultáneos $(3,17)$ y en alguna ocasión unicamente se ha identificado el microorganismo en las lesiones y no en los hemocultivos (2, 23). En nuestra casuística, el microorganismo se aisló tanto en los hemocultivos como en los nódulos de Osler. La tinción de Gram de la muestra obtenida por punción aspirativa del nódulo de Osler, permitió llegar a un diagnóstico aproximativo del microorganismo responsable de la endocarditis lo que ayudó a instaurar el tratamiento antibiótico más adecuadamente. Otro aspecto que destacamos, es que los nódulos de Osler sólo se observaron en las endocarditis que afectaban a las válvulas izquierdas y no a las derechas, lo que apoya el origen embolígeno de los nódulos de Osler.

A raíz de nuestra experiencia recomendamos que cuando se detecten nódulos de Osler se tome precozmente una muestra por punción-aspiración y se proceda a realizar una tinción de Gram y cultivo de la misma, ello puede dar una información rápida y precisa de la etiología de la EI en los UDVP.

\section{Bibliografía}

1. Scheld WM, Sande MA. Endocarditis and intravascular infections. En: Mandell GL, Bennett JE, Dolin R, eds; Mandell, Douglas and Bennett's Principles and practice of Infectious Diseases. Nueva York: Churchill Livingstone; 1995: 740-83.

2. Cardullo AC, Silvers DN, Grossman ME. Janeway lesions and Osler's nodes: a review of histopathologic findings. J Am Acad Dermatol 1990; 22: 1088-90.

3. Parikh SK, Lieberman A, Colbert DA, Silvers DN, Grossman ME. The identification of methicillin-resistant Staphylococcus aureus in Osler's nodes and Janewey lesions of acute bacterial endocarditis. J Am Acad Dermatol 1996; 35: 767-8.

4. Durack DT, Lukes AS, Bright DK, Duke Endocarditis Service. New criteria for diagnosis of infective endocarditis: utilization of specific echocardiographic findings. Am J Med 1994; 96: 200-9.

5. Von Reyn CF, Levy BS, Arbeit RD, Friedland G, Crumpacker CS Infective endocarditis: an analysis based on strict case definitions. Ann Intern Med 1991; 94: 505-18

6. Watanakunakorn C. Staphylococcus aureus endocarditis at a community teaching Hospital, 1980 to 1991. An analysis of 106 cases. Arch Intern Med 1994; 154: 2330-5.

7. Carton JA, Asensi V, Maradona JA, Segovia E, Simarro C, Pérez González F et al. Endocarditis infecciosa sobre válvula natural: perfil epidemiológico y análisis de la mortalidad entre los años 1984 y 1993. Med Clin (Barc) 1995; 104: 493-9.

8. Siddiq S, Missri J, Silverman DI. Endocarditis in an Urban Hospital in the 1990s. Arch Intern Med 1996; 156: 2454-8.

9. Lerner PI, Weinstein I. Infectious endocarditis in the antibiotic era. N Engl J Med 1966; 274: 199-206, 256-66, 323-31, 389-99.

10. Hermans PE. The clinical manifestations of infective endocarditis. Mayo Clin Proc 1982; 57: 15-21.
11. Farrior JB III, Silverman ME. A consideration of the differences between a Janeway's lesion and an Osler's node in infectious endocarditis. Chest 1976; 70: 239-43.

12. Osler W. The chronic intermittent fever of endocarditis. Practitioner 1893; 51: 181-90.

13. Merklen P, Wolf M. Parcipation des endothéliities arteriocapillaries au syndrome del'endocardite maligne lente. Press Med 1928; 36: 97100

14. Lian C, Nicolau S, Poincloux P. Histophatologie de nodule d'Osler: étude sur l'endoth'eliite de l'endocardite maligne a évolution lente. Press Med 1929; 37: 497-9.

15. Cornil L, Mosinger M, Jouve AX. Contribution a l'etude histologique du nodule d'Osler. Ann Anat Pathol 1936; 13: 675-86.

16. Von Germminger G, Winkelman RK. Osler's node of subacute bacterial endocarditis. Arch Dermatol 1967; 95: 91-4.

17. Alpert JS, Krous HF, Dalen JE, O'Rourke, Bloor CM. Pathogenesis of Osler's nodes. Ann Intern Med 1976; 85: 471-3.

18. Kerr A, Jr, Tan JS. Biopsies of the Janeway lesion of infective endocarditis. J Cutan Pathol 1979; 6: 124-9.

19. Dusky BM. Recurrent Osler's nodes in systemic lupus erythematosus. Angiology 1969; 20: 33-7.

20. Puklin JE, Balis GA, Bentley DW. Culture of an Osler's node. A diagnostic tool. Arch Intern Med 1971; 127: 296-8.

21. Mennecier D, Debien B, Dupeyron C, Pats B, Rouvier B. Faux panaris d'Osler. Press Med 1998; 27: 786.

22. Dumont D, Mathieu D, Alemanni M, Eb F, Manigand G. Endocardite d'Osler probablement due á Chlamydia pneumoniae (souche TWAR). Press Med 1990; 19: 1054.

23. Yee J, McAllister K. The utility of Osler's nodes in the diagnosis of infective endocarditis. Chest 1987; 94: 751-2. 